\title{
Enhancement of flow anisotropies due to magnetic field in relativistic heavy-ion collisions
}

\author{
Ranjita K. Mohapatra, P. S. Saumia, and Ajit M. Srivastava \\ Institute of Physics, Sachivalaya Marg, Bhubaneswar 751005, India
}

\begin{abstract}
It is known that the presence of background magnetic field in cosmic plasma distorts the acoustic peaks in CMBR. This primarily results from different types of waves in the plasma with velocities depending on the angle between the magnetic field and the wave vector. We consider the consequences of these effects in relativistic heavy-ion collisions where very strong magnetic fields arise during early stages of the plasma evolution. We show that flow coefficients can be significantly affected by these effects when the magnetic field remains strong during early stages due to strong induced fields in the conducting plasma. In particular, the presence of magnetic field can lead to enhancement in the elliptic flow coefficient $v_{2}$

PACS numbers: 52.35.Bj, 25.75.Ld, 12.38.Mh
\end{abstract}

It has been recently shown by us that a deep correspondence exists between the physics of inflationary density fluctuations in the early universe which result in the CMBR acoustic peaks and the physics of flow in relativistic heavy-ion collision experiments (RHICE) [1]. We further showed that important features such as acoustic peaks and suppression of superhorizon fluctuations may be present in a plot of root-mean square values of flow coefficients $v_{n}^{r m s}$. The possibility of presence of this suppression of superhorizon fluctuations has been recently pointed out by Sorensen in RHIC data 2]. There have been several works now which discuss flow fluctuations for a large range of values of $n$ [3]. This non-trivial connection between the superhorizon fluctuations of inflationary universe and similar fluctuations in RHICE is now discussed in several works and its various consequences are explored (see, e.g. [4]). We mention that such a connection between physics of RHICE and that of inflationary universe was never anticipated earlier, and indeed, at first sight, it looks surprising that a concept like superhorizon fluctuation which arises from highly non-trivial, superluminal expansion phase of the very early universe could have any relevance for relativistic heavyion collision experiments in laboratory. Such superhorizon fluctuations in RHICE originate from the fact that in the center of mass frame the thermalization (and any local homogenization) happens rather quickly, within about $1 \mathrm{fm}$. Initial parton energy density distribution from HIJING shows that transverse fluctuations (arising from localization of partons inside initial nucleons, and from the fluctuations in nucleon coordinates) with wavelengths significantly larger than $1 \mathrm{fm}$ are necessarily present at the time $1 \mathrm{fm}$ even in central collisions.

It was also emphasized in [1] that various analysis tools of CMBR anisotropies can be effectively utilized for RHICE. It was proposed in [1] that instead of focusing on the average values of the flow coefficients $v_{n}$ for small values of $n$ [5], one should calculate root-mean square values of the flow coefficients $v_{n}^{r m s}$ for a large range of $n$ upto 30-40. Further, these calculations should be performed in a lab fixed frame, which eliminates the difficulties associated with determination of event plane for conventional elliptic flow analysis for non-central collisions. It was shown in [1] that a plot of values of $v_{n}^{r m s}$ vs. $n$ can be used for directly probing various flow coefficients, in particular, the elliptic flow for non-central collisions [ $[$ ], without any need for the determination of event-plane.

In this paper we continue to explore this fertile connection between CMBR physics and RHICE. It was shown in ref. [7] that the presence of background magnetic field in cosmic plasma can distort the acoustic peaks in CMBR. This happens due to the presence of different waves in the plasma, with velocities depending on the angle between the magnetic field and the wave vector. Presence of very strong magnetic fields in the plasma (of order $10^{15}$ Tesla) during early stages in RHICE has been explored extensively recently in connection with the exciting possibility of observing CP violation effects [8-10]. An important effect of the presence of such strong magnetic fields in the plasma will be to lead to strong variations in velocities of different types of waves in the plasma. In particular the velocity varies with the angle between the wave vector and the direction of the magnetic field. It is thus obvious that this may qualitatively affect the development of anisotropic flow. We argue that the flow coefficients can be significantly affected by these effects. In particular, the presence of magnetic field can lead to enhancement in the elliptic flow coefficient $v_{2}$ by almost $30 \%$. (Note, we use $v_{2}$ with the present definitions to denote the elliptic flow even though we do not adopt the conventional usage of the eccentricity for defining the corresponding spatial anisotropy.) This raises the interesting possibility whether a larger value of $\eta / s$ can be accommodated by RHIC data when these effects are incorporated using full magnetohydrodynamical simulations.

An important issue here is the time scale over which the magnetic field remains strong. The magnetic field arising from the valence charges of the initial nuclei peaks to strong values for a very short time, essentially the passing time of the Lorentz contracted nuclei $\left(\sim 0.2 \mathrm{fm}\right.$ for RHIC energies). Subsequently it rapidly decays $\left(\sim \tau^{-2}\right)[9]$. In such a situation the effect of magnetic field on flow coefficient will be suppressed as time scale for the development of flow 
is several fm. Even at LHC, where expected magnetic field is more than an order of magnitude larger than that at RHIC, the effect of this initial pulse of magnetic field on flow coefficients may not be very significant, though due to various uncertainties, a full magnetohydrodynamics simulation is needed to investigate these issues.

However, it has been recently pointed out [11] that magnetic fields of similar magnitude, as the peak value of the external field, can arise from induced currents due to rapidly decreasing external field. Further, in the quasi-static approximation, the magnetic field satisfies a diffusion equation with the diffusion constant equal to $1 /(\sigma \mu)$ where $\mu$ is the magnetic permeability and $\sigma$ is the electrical conductivity [12]. It is argued in ref. [11] that due to this the magnetic field may survive for much longer time, and can lead to interesting effects.

We will take $\mu \sim 1$ (refs. 11, 12]). However, the value of electrical conductivity we take is smaller than what is taken in ref.[11]. We use $\sigma \simeq 0.3 T\left(=0.3 \mathrm{fm}^{-1}\right.$ for $\left.\mathrm{T} \simeq 200 \mathrm{MeV}\right)$ from refs. 13]. The time scale $\tau$ over which the magnetic field remains essentially constant [11] over length scale $L$ is, $\tau \simeq L^{2} \sigma / 4$. For $L=5-6 \mathrm{fm}$, we get $\tau \simeq 2-3$ $\mathrm{fm}$. For higher temperatures $\sigma$ will be larger increasing the value of $\tau$. (See, also, ref. [14] for effects of leptons on $\sigma$, though for RHICE this may not be significant.) $\sigma$ is also expected to increase due to the effects of magnetic field in the plasma [15], further increasing the value of $\tau$.

It is important to note that the initial magnetic field will enter the medium in the longitudinal direction as the medium is only about $1 \mathrm{fm}$ thick in that direction at the initial stage. For this the relevant quantity is the penetration depth $\delta \sim(\mu \sigma \omega)^{-1 / 2}$ where $\omega$ is the angular frequency of electromagnetic wave. Initial magnetic field, being a narrow pulse of time duration $t \simeq 0.2 \mathrm{fm}$ (typically the width of Lorentz contracted Nuclei, for RHIC energies), can be taken to have $\omega \simeq 30 \mathrm{fm}^{-1}$. This gives the penetration depth of order $3 \mathrm{fm}$. Thus, the picture of magnetic field diffusing through the entire region of the plasma with typical length scale of several $\mathrm{fm}$, and lasting with high initial peak values for time scales of several fm, is self consistent. (Though, note that the time scale of the decay of the field being of same order as the system size makes the assumption of quasi-static field, and to that extent the assumption of ideal magnetohydrodynamics, only marginally valid.) As significant flow anisotropy develops also in the time scale of order 3-4 fm [16], it is then reasonable to assume that magnetic field can be taken to be almost constant for this duration of flow development. In conclusion, induced magnetic field, with similar magnitude as the peak value of the initial magnetic field pulse, decays slowly and, for time scales relevant for our model, can be taken to be approximately constant.

With this discussion, we continue to investigate the effects of (an approximately constant) magnetic field on sound waves in QGP produced in RHICE. For the non-relativistic plasma the effect of magnetic field can be simply described in terms of three different waves [7, 17]. There is a fast magnetosonic wave which, for small magnetic fields, becomes the ordinary sound wave. Its velocity is given by $c_{+}^{2}=c_{s}^{2}+v_{A}^{2} \sin ^{2} \theta$, where $v_{A}=B_{0} / \sqrt{4 \pi \rho}$ is the Alfvén velocity and $\theta$ is the angle between the wave vector and the magnetic field $\mathbf{B}_{0} . c_{s}$ is the sound velocity and $\rho$ is the plasma density. Slow magnetosonic wave has velocity $c_{-}^{2}=v_{A}^{2} \cos ^{2} \theta$, and the Alfvén wave velocity is given by $c_{A}^{2}=v_{A}^{2} \cos ^{2} \theta$. These expressions for magnetosonic wave velocities are valid for the case when $v_{A}<<c_{s}$. It was argued in [7] that fast magnetosonic waves lead to distortion of CMBR acoustic peaks while the slow magnetosonic waves may lead to long period modulation of the peaks.

For the situation of plasma in RHICE one needs to consider the situation of relativistic magnetohydrodynamics. We will assume the applicability of magnetohydrodynamical description for the plasma at RHICE and restrict our consideration to wavelengths much larger than the Debye screening length. For the relativistic case, the expressions for the velocities of these waves are given below (we use natural units with $c=1$ ). For the following discussion, we have followed ref. [17].

\section{Alfvén waves}

Phase velocity $\mathbf{v}_{p h}$ and group velocity $\mathbf{v}_{g r}$ of these waves are

$$
\mathbf{v}_{p h}=\frac{B_{0} \cos \theta}{\sqrt{\omega_{0}}} \mathbf{n}, \quad \mathbf{v}_{g r}=\frac{\mathbf{B}_{\mathbf{0}}}{\sqrt{\omega_{0}}},
$$

where $\mathbf{n}=\mathbf{k} / k, \mathbf{k}$ is the wave vector and $\theta$ is the angle between $\mathbf{n}$ and the magnetic field $\mathbf{B}_{\mathbf{0}} . \omega_{0}=\rho_{0} h_{0}+B_{0}^{2}$ where $h$ is the specific enthalpy (subscript denoting the background values) defined as [17] $\rho h \equiv \rho c^{2}+\rho \epsilon+P$. Here, $\rho \epsilon$ is the internal energy, $\rho c^{2}$ is the rest mass energy and $P$ is the pressure. For ultra-relativistic case we take $\rho h=4 P$.

\section{Magnetosonic waves}

These are the waves which are relevant for our case of discussion of flow as they involve density perturbations. Phase velocities for these waves are given by

$$
\mathbf{v}_{p h}=v_{p h} \mathbf{n}=\mathbf{n}\left(\frac{1}{2}\left[\left(\rho_{0} h_{0} / \omega_{0}\right) c_{s}^{2}+v_{A}^{2}\right]\right)^{1 / 2}\left(1+\delta \cos ^{2} \theta \pm a\right)^{1 / 2} .
$$




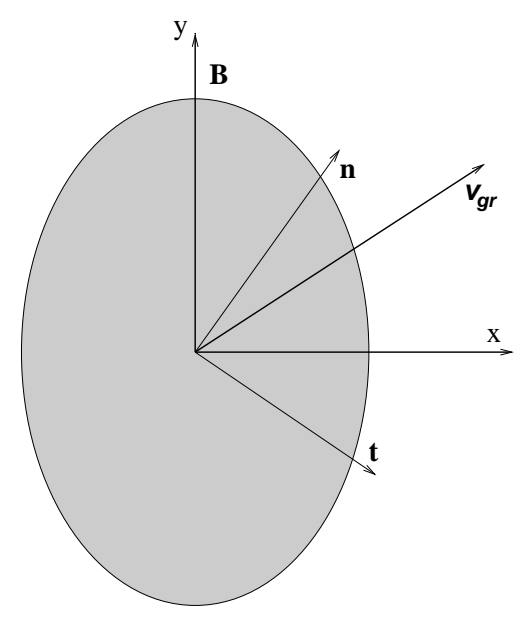

FIG. 1:

Typical situation expected in RHICE. Magnetic field points in y direction and the direction of the group velocity $\mathbf{v}_{g r}$ is obtained from $\mathbf{n}$ and $\mathbf{t}$ via Eq.(5).

Here + and - signs correspond to the fast and slow magnetosonic waves respectively, $v_{A}=B_{0} / \sqrt{\omega_{0}}$ is the Alfvén speed, and $\delta$ and $a$ are defined below.

$$
\begin{gathered}
a^{2}=\left(1+\delta \cos ^{2} \theta\right)^{2}-\sigma \cos ^{2} \theta \\
\delta=\frac{c_{s}^{2} v_{A}^{2}}{\left[\left(\rho_{0} h_{0} / \omega_{0}\right) c_{s}^{2}+v_{A}^{2}\right]}, \quad \sigma=\frac{4 c_{s}^{2} v_{A}^{2}}{\left[\left(\rho_{0} h_{0} / \omega_{0}\right) c_{s}^{2}+v_{A}^{2}\right]^{2}} .
\end{gathered}
$$

( $\sigma$ here should not be confused with the conductivity discussed above.) For propagation of density perturbations, as relevant for the evolution of flow anisotropies, the relevant wave velocity is the group velocity for the magnetosonic waves,

$$
\mathbf{v}_{g r}=v_{p h}\left[\mathbf{n} \pm \mathbf{t} \frac{\left[\sigma \mp 2 \delta\left(a \pm\left(1+\delta \cos ^{2} \theta\right)\right)\right] \sin \theta \cos \theta}{2\left(1+\delta \cos ^{2} \theta \pm a\right) a}\right]
$$

Here $\mathbf{t}=\left[\left(\mathbf{B}_{\mathbf{0}} / B_{0}\right) \times \mathbf{n}\right] \times \mathbf{n}$, and again the upper and lower signs $( \pm$ or $\mp)$ correspond to the fast and the slow magnetosonic waves respectively. For a given magnetic field $\mathbf{B}_{\mathbf{0}}$, the direction of $\mathbf{n}$ can be varied to generate group velocities of these waves in different directions. Fig.1 shows a typical situation of various vectors in Eq.(5) expected in RHICE. It is important to note that the direction of $\mathbf{v}_{g r}$ depends on the relative factors multiplying $\mathbf{n}$ and $\mathbf{t}$ in Eq.(5). This in turn depends on properties of the plasma like energy density. Thus due to the presence of spatial gradients in RHICE, even along a fixed azimuthal direction, we will expect the direction of $\mathbf{v}_{g r}$ to keep varying with the radial distance. This can lead to the development of very complex flow patterns, possibly leading to generation of vorticity.

For the strength of the magnetic field expected in RHICE, we use the estimates given in refs. [9, 10] for $\mathrm{Au}-\mathrm{Au}$ collisions at RHIC. For first estimates, we use a simple parametrization and take the magnetic field to be proportional to the impact parameter (with somewhat larger magnitudes than in ref. [9, 10] to illustrate the effects).

$$
B_{0}=10^{5} \frac{b(f m)}{10} \mathrm{MeV}^{2}
$$

This corresponds to the maximum magnetic field of about $10^{15}$ Tesla and the scale of $10 \mathrm{fm}$ is taken from [10] (where time integrated value of $e B_{0}$ is calculated, as coupling to charge particles is of interest there) as the approximate limiting value of the impact parameter $b$ up to which proportionality with $b$ is expected. The effect of magnetic field on wave propagation here comes from an effective magnetic pressure arising from the freezing of magnetic field lines in the plasma in the magnetohydrodynamic limit. Note that the importance of charges (quarks) here is only in 
establishing the magnetohydrodynamic conditions such that the electric field in the comoving frame vanishes. There is no direct pressure generated by interaction of magnetic field with quarks. (Thus it is of no relevance that gluons, which do not interact with the magnetic field, dominate the energy density of the plasma.) The distortions of magnetic field lines in the presence of density perturbations cost energy leading to an extra contribution to pressure from the presence of magnetic field. This is what is responsible for increasing the effective sound speed as given above.

We will assume a magnetic field with magnitude $B_{0}$ given above which is uniform over the region of the plasma, and as we have discussed above, is approximately constant for the early stages of time scale of few fm. This early time duration is important for the evolution of flow anisotropies [16]. The magnetic field has direction in the transverse plane, normal to the direction of the impact parameter. From the expression of $\mathbf{v}_{g r}$ in Eq.(5) we see that an important factor is the ratio $B_{0}^{2} / P$ where $P$ is the pressure of the plasma which we take to be the quark-gluon plasma with two light flavors with the pressure given by $P=\frac{37}{90} \pi^{2} T^{4}$. Again, to illustrate the effects of the magnetic field on flow, we consider the situation at a lower value of the temperature $T=180 \mathrm{MeV}$. As the important effects occur for strong magnetic field which occurs for large impact parameter, a lower value of $T$ (compared to what is expected in central collisions) may not be very unreasonable. We are considering the effects of magnetic field relevant for Au-Au collisions at RHIC on somewhat larger side of the estimates. However, for higher energy collisions, e.g. at LHC, larger values of $B_{0}$ (by an order of magnitude compared to RHIC) should be routinely expected, see e.g. [18].

We follow the procedure described in [1] for calculating $v_{n}^{r m s}$ using HIJING [19]. We start with the initial transverse energy density $\epsilon_{t r}$ distribution for $\mathrm{Au}-\mathrm{Au}$ collision at $200 \mathrm{GeV} / \mathrm{A}$ center of mass energy from HIJING. For details, see ref. [1]. We assume that the hydrodynamic description becomes applicable by $\tau=\tau_{e q}$, which we take to be $1 \mathrm{fm}$ and calculate the anisotropies in the fluctuations in the spatial extent $R(\phi)$ at this stage, where $R(\phi)$ represents $\epsilon_{t r}$ weighted average of the transverse radial coordinate in the angular bin at azimuthal coordinate $\phi$. As emphasized above, angle $\phi$ is taken in a lab fixed coordinate frame. We divide the region in 50 - 100 bins of azimuthal angle $\phi$, and calculate the Fourier coefficients of the anisotropies in $\delta R / R \equiv(\bar{R}-R(\phi)) / \bar{R}$ where $\bar{R}$ is the angular average of $R(\phi)$. Note that in this way we are representing all fluctuations essentially in terms of fluctuations in the boundary of the initial region. We use $F_{n}$ to denote Fourier coefficients for these spatial anisotropies, and use $v_{n}$ to denote $n_{t h}$ Fourier coefficient of expected momentum anisotropy in $\delta p / p$ defined in the lab frame. We have generated events using HIJING and we present sample results for $\mathrm{Au}-\mathrm{Au}$ collision at $200 \mathrm{GeV} / \mathrm{A}$ center of mass energy. In all the plots, the averages are taken over 1000 events.

In [1], the root mean square values $v_{n}^{r m s}$ of the flow Fourier coefficients were obtained from spatial $F_{n} \mathrm{~s}$ simply by using proportionality factor of 0.2 (with a minus sign as $F_{n}$ will be negative). We include the effect of magnetic field and the resulting angle dependent velocity of the (fast) magnetosonic wave by replacing this proportionality factor to $0.346 \times \mathbf{v}_{g r}$. Here the group velocity of the (fast) magnetosonic wave $\mathbf{v}_{g r}$ (Eq. (5)) changes with the angle in the event plane. The assumption here is that the flow coefficients are proportional to the sound velocity [16]. (Ignoring that now the time scale of the development of flow may also vary with the azimuthal angle). The factor of 0.346 is chosen so that the proportionality constant becomes 0.2 for zero magnetic field case with the usual sound velocity $c_{s}=1 / \sqrt{3}$.

In Fig.2 we show the plots of $v_{n}^{r m s}$ for different values of impact parameter $b$ with magnetic field given by Eq.(6) (Solid curves) and without magnetic field (dashed curves). These plots show that magnetic field can strongly affect values of $v_{n}^{r m s}$.

We have also calculated average values of flow coefficients in the event plane. Fig.3 shows the plots of the average flow coefficients $v_{n}$ for $b=10 \mathrm{fm}$. Solid curve shows the plot with the presence of magnetic field and the dashed curve shows the plot in its absence. Note that both the curves approach almost zero value beyond $n \simeq 10$ (as higher $v_{n} \mathrm{~s}$ lose correlation with the event plane).

It is important to study the effect of magnetic field on the elliptic flow $v_{2}$. In Fig. 4 we show the plot of the ratio of $v_{2}$ with and without the magnetic field, i.e. $v_{2}(B) / v_{2}(0)$ as a function of the impact parameter $b$. Elliptic flow itself varies with $b$ and this ratio helps us in separating the effect of the magnetic field on $v_{2}$ as the field varies with $b$. Note that this ratio becomes as large as 1.3 for $b=10 \mathrm{fm}$ (for which the magnetic field takes its largest value in Eq.(6)). This strong enhancement in the value of $v_{2}$ is important especially as it raises the interesting possibility whether a larger value of $\eta / s$ can be accommodated by RHIC data when proper accounts of magnetohydrodynamics are incorporated in flow calculations.

Due to anisotropy of magnetosonic wave velocity, the radial flow itself will get modified. To incorporate this effect we modulated the initial spatial profile with a suitable weight factor proportional to $\mathbf{v}_{g r}$. However, its effects were insignificant. It still remains a possibility that for much stronger magnetic fields non-trivial flow anisotropies may arise even with almost isotropic initial conditions (though, for central collisions one expects very small magnetic fields). Also, note that the direction of $\mathbf{v}_{g r}$ depends on the relative weights of factors multiplying $\mathbf{n}$ and $\mathbf{t}$ in Eq.(5) which depends on quantities like the plasma energy density etc. Thus due to the presence of spatial gradients of plasma density in RHICE, even along a given azimuthal direction in the transverse plane the direction of $\mathbf{v}_{g r}$ will keep changing. In particular, fluctuations of energy density will lead to fluctuations in $\mathbf{v}_{g r}$ as well. Clearly due to such 

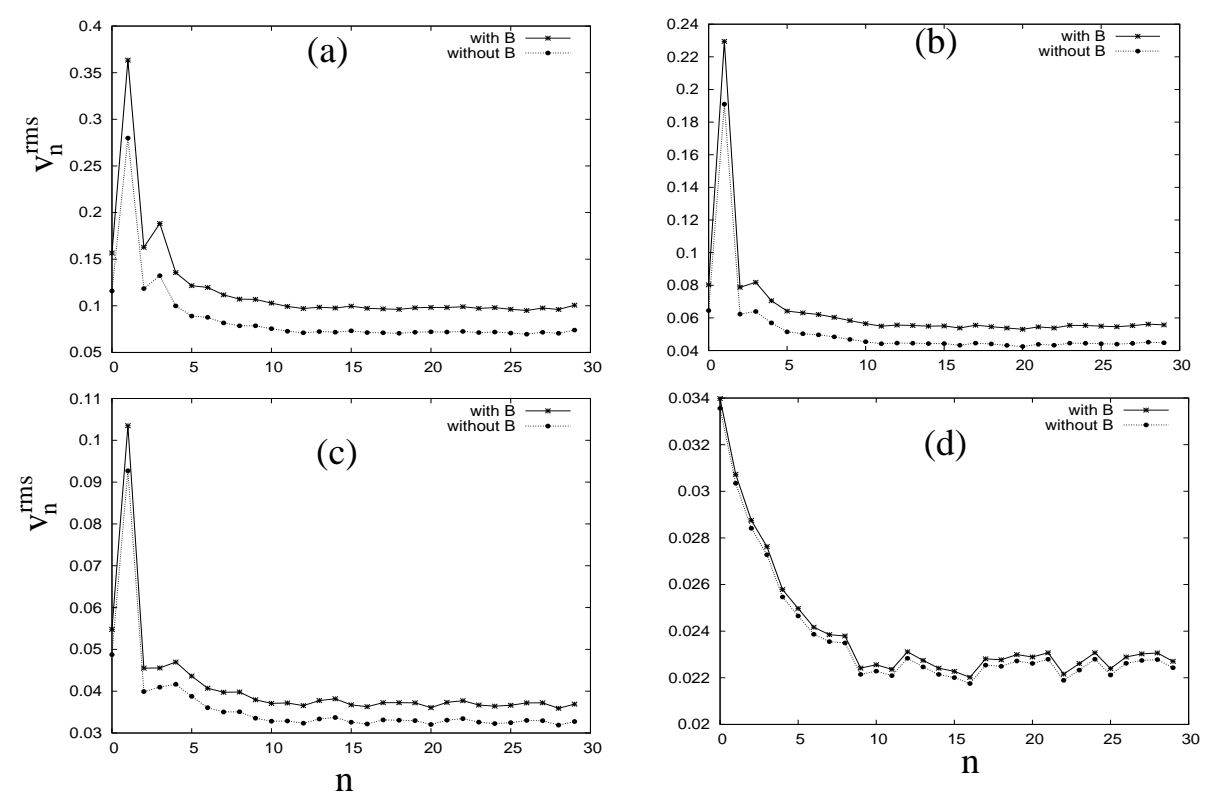

FIG. 2:

Plots of $v_{n}^{r m s}$ for different values of impact parameters $b$. Solid (dashed) curves show the plots in the presence (absence) of magnetic field. (a),(b),(c),(d) correspond to the values of $b=10,8,6,2 \mathrm{fm}$ respectively.

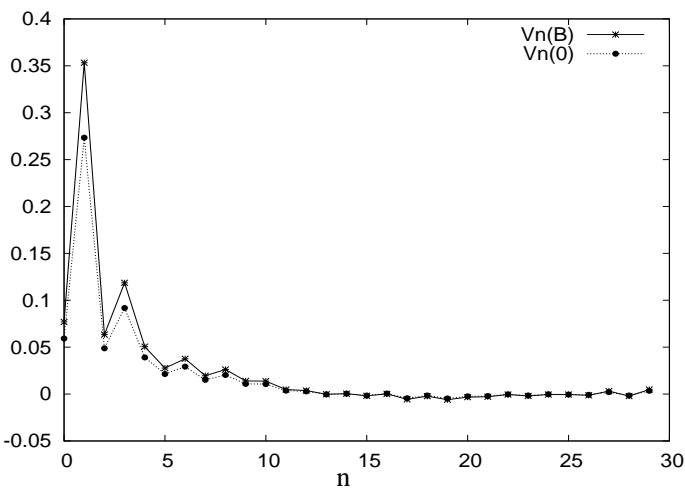

FIG. 3:

Plots of the average flow coefficients $v_{n}$ for $b=10 \mathrm{fm}$. Solid curve shows the plot with the presence of magnetic field and the dashed curve shows the plot in its absence.

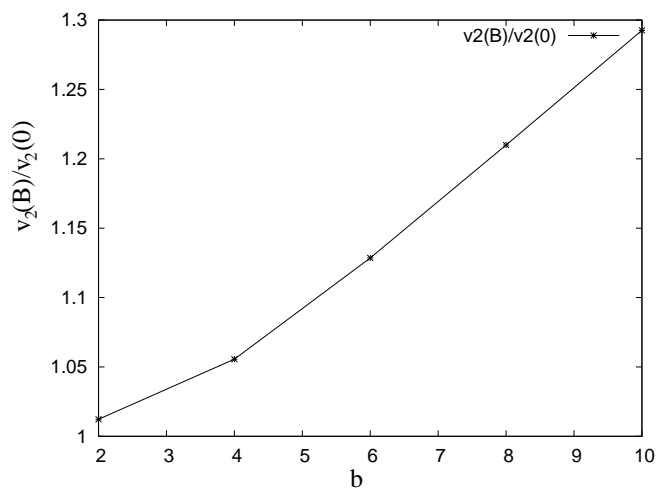

FIG. 4:

Plot of the ratio of $v_{2}$ with and without the magnetic field, as a function of the impact parameter $b$ (in fm). 
phenomena one expects a complex pattern of flow, even possibly leading to vorticity, to develop in RHICE than just radial flow and flow anisotropies. We have also checked the effects of magnetic field on other features of the plots of $v_{n}^{r m s}$ discussed in [1], in particular on the acoustic peaks. The effects are similar to what is shown in Fig.2 hence we do not show it here. It remains to be explored how the slow magnetosonic waves affect various features of the plots of $v_{n}^{r m s}$ in view of its proposed long period modulation of the acoustic peaks in CMBR. The role of Alfvén waves also needs to be explored in the evolution of various fluctuations in RHICE (especially in view of their effects on CMBR 20]). Our approach in this work has been to present rough estimates of various possible effects of the presence of the magnetic field in RHICE. Detailed magnetohydrodynamical simulations are needed to probe these effects. Especially exciting will be the possibility of large effects at LHC energies where large magnetic fields (by almost an order of magnitude compared to RHIC) are expected [18].

We are very grateful to Abhishek Atreya, Anjishnu Sarkar, Uma Shankar Gupta, and Trilochan Bagarti for useful discussions.

[1] A. P. Mishra, R. K. Mohapatra, P. S. Saumia, and A. M. Srivastava, Phys. Rev. C 77, 064902 (2008); Phys. Rev. C 81, 034903 (2010).

[2] P. Sorensen, Proc. 24th Winter Workshop on Nuclear Dynamics, 2008, arXiv:0808.0503.

[3] P. Sorensen, arXiv:0905.0174 [nucl-th]; J. Phys. G37, 094011 (2010); B. Alver and G. Roland, Phys. Rev. C 81, 054905 (2010); B.H. Alver, C. Gombeaud, M. Luzum, and J.-Y. Ollitrault, Phys. Rev. C82, 034913 (2010);

[4] P. Staig and E. Shuryak, arXiv:1008.3139 [nucl-th]

[5] P. Sorensen for the STAR Collaboration, J. Phys. G 34, S897 (2007); J. Phys. G 35, 104102, (2008); R.S. Bhalerao and J.-Y. Ollitrault, Phys. Lett. B 641, 260 (2006); S. Mrowczynski and E. Shuryak, Acta Phys. Polon. B 34, 4241 (2003).

[6] J.-Y. Ollitrault, Phys. Rev. D 46, 229 (1992); S. Voloshin and Y. Zhang, Z. Physik C70, 665 (1996); S.A. Volosin, A.M. Poskanzer, and R. Snellings, arXiv:0809.2949

[7] J. Adams, U.H. Danielsson, D. Grasso, and H. Rubinstein, Phys. Lett. B 388, 253 (1996).

[8] D. Kharzeev, R.D. Pisarski, and M.H.G. Tytgat, Phys. Rev. Lett. 81, 512 (1998), S.A. Voloshin, Phys. Rev. C 70, 057901 (2004).

[9] D.E. Kharzeev, L.D. McLerran, and H.J. Warringa, Nucl. Phys. A 03227 (2008)

[10] M. Asakawa, A. Majumder, and B. Muller, Phys. Rev. C 81, 064912 (2010).

[11] K. Tuchin, arXiv:1008.1604; Phys. Rev. C 82, 034904 (2010).

[12] L. Landau and E. Lifshitz, "Electrodynamics of Continuous Media", (Pergamon Press, N.Y., USA, 1984), Sect. 58; J.D. Jackson, "Classical Electrodynamics", 3rd Edition, John Wiley \& Sons, Inc., USA, 1999, Sect. 5.18.

[13] S. Gupta, Phys. Lett. B 597, 57 (2004); H.-T. Ding et al. arXiv:1012.4963

[14] P.B. Arnold, G.D. Moore, and L.G. Yaffe, JHEP 0305, 051 (2003).

[15] P.V. Buividovich, et al. Phys. Rev. Lett. 105, 132001 (2010).

[16] J.-Y. Ollitrault, Eur. J. Phys. 29, 275 (2008); R. S. Bhalerao, J. P. Blaizot, N. Borghini, and J.-Y. Ollitrault, Phys. Lett. B 627, 49 (2005).

[17] "Advanced Magnetohydrodynamics", J.P. (Hans) Goedbloed, R. Keppens, and S. Poedts, (Cambridge University Press, UK, 2010)

[18] V. Skokov, A.Yu. Illarionov, and V. Toneev, Int. J. Mod. Phys. A 24, 5925 (2009).

[19] X.N. Wang and M. Gyulassy, Phys. Rev. D 44, 3501 (1991); Comput. Phys. Commun. 83, 307 (1994).

[20] J. Kim and P. Naselsky, JCAP 0907, 041 (2009); G. Chen et al. Astrophys. J. 611, 655 (2004). 